\title{
ФИЗИКО-МАТЕМАТИЧЕСКИЕ НАУКИ
}

\author{
ПОВЫШЕНИЕ ЭФФЕКТИВНОСТИ ОБУЧЕНИЯ КУРСАНТОВ МАТЕМАТИЧЕСКИМ \\ АЛГОРИТМАМ, ПРЕДСТАВЛЯЕМЫМ В ТАБЛИЧНОЙ ФОРМЕ
}

Париин Анатолий Васильевич кандидат физ.-мат. наук, профессор ВУНЦ ВВС «Военно-воздушная академия им. проф. Н.Е. Жуковского и Ю.А. Гагарина», 2. Воронеж

\section{INCREASING THE EFFECTIVENESS OF TEACHING CURSORS MATHEMATICAL ALGORITHMS, PRESENTED IN TABLE FORM}

\author{
Anatoly Vasilievich Parshin \\ candidate of physical and mathematical sciences, professor \\ Military Research Center of the Air Force «Air Force Academy named after \\ prof. N.E. Zhukovsky and Yu.A. Gagarin»Voronezh \\ DOI: $10.31618 /$ nas.2413-5291.2020.3.55.225
}

\section{Аннотация}

В дисциплине «Математика», преподаваемой в инженерно-технических вузах, выделяется ряд математических алгоритмов, отличительной особенностью которых является табличная форма их представления. Они в основном составляют содержание раздела «Линейная алгебра». Автоматизация этой формы средствами табличного программного продукта MS Excel путем проведения занятия с использованием ПЭВМ, дополнительно объединенных в видеосеть, приводит к повышению эффективности обучения курсантов вычислению этих алгоритмов.

\section{Abstract}

In the discipline "Mathematics", taught in engineering universities, a number of mathematical algorithms are distinguished, the distinguishing feature of which is the tabular form of their presentation. They basically make up the content of the Linear Algebra section. Automation of this form by means of the MS Excel spreadsheet software by conducting classes using personal computers, additionally combined into a video network, leads to an increase in the effectiveness of teaching cadets how to calculate these algorithms.

Ключевые слова: табличная форма представления математических алгоритмов; табличный процессор MS Excel; ПЭВМ; видеосеть; эффективность.

Keywords: tabular presentation of mathematical algorithms; MS Excel spreadsheet; PC; video network; efficiency.

При изучении линейной алгебры в военных инженерно-технических вузах одно из первых занятий отводится на освоение методов (алгоритмов) вычисления определителей. От того насколько успешно удается преподавателю реализовать дидактические цели этого занятия, во многом зависит и эффективность последующих практических занятий. Действительно, задача вычисления определителя возникает как «подзадача» при вычислении векторного и смешанного произведения векторов, при решении систем линейных алгебраических уравнений, при вычислении обратных матриц и во многих других более содержательных задачах линейной алгебры.

Обычно на освоение обсуждаемого математического аппарата отводится одно двухчасовое практическое занятие «Вычисление определителей», включающее следующие учебные вопросы:

1. Вычисление определителей второго и третьего порядков.

2. Вычисление определителей $n$-го порядка.

Отметим, что определитель - это некоторое число, вычисляемое по тем или иным правилам. Поэтому решение учебных задач по нахождению определителей необходимо доводить до конечного числового результата, проводя поэтапно все необходимые промежуточные расчеты. В связи с этим особенностью обсуждаемого занятия является его насыщенность действиями, отвлекающими внимание курсантов от изучаемого математического аппарата (помеховые или рутинные вычисления). Эта особенность занятия, а также табличная форма осваиваемых на нем алгоритмов, наряду с педагогической интуицией, позволяют выдвинуть гипотезу о том, что автоматизация рутинных вычислений средствами табличного программного продукта MS Excel путем проведения занятия с использованием ПЭВМ, дополнительно объединенных в видеосеть [1], приведет $\kappa$ повышению эффективности обучения курсантов вычислению определителей.

В данной статье приводятся результаты педагогического исследования, посвященного проверке этой гипотезы, включая сравнительный анализ возможностей конкурирующих программных продуктов MS Excel и Derive 6 [2] по интенсификации проведения обсуждаемого занятия. 
Исследование

экспериментальным Военного авиационного инженерного университета (г. Воронеж). Следует отметить, что обсуждаемому практическому занятию предшествовали две вводные лабораторные работы вычислительного практикума, на которых курсанты имели возможность приобрести первичные умения работы с табличным редактором MS Excel и компьютерной математической системой Derive 6 [3].

Педагогический эксперимент согласно требованиям современной педагогики [4] проводился в три этапа. На начальном этапе (констатирующий эксперимент) экспертом был проведен сравнительный анализ затрат времени на решение комплекта задач (по теме рассматриваемого занятия) тремя способами:

1) вручную (с использованием в необходимых случаях калькулятора);

1) с использованием компьютерной математической системы Derive 6;

2) с использованием электронных таблиц MS Excel;

Комплект состоял из 14 заданий.

Результаты первого этапа эксперимента приведены в таблице 1 и на рисунке 1.

Таблица 1

Время, затраченное экспертом на решение комплекта задач

\begin{tabular}{|c|c|c|c|c|c|}
\hline $\begin{array}{c}\text { № } \\
\text { задачи }\end{array}$ & $t_{B}$, Мин & $t_{D}$, Мин & $t_{E}$, Мин & $\frac{t_{D}-t_{E}}{t_{E}} \cdot 100 \%$ & $\frac{t_{B}-t_{E}}{t_{E}} \cdot 100 \%$ \\
\hline 1.1 & 0.8 & 1.0 & 1.0 & 0 & -20 \\
\hline 1.2 & 1.1 & 0.9 & 1.1 & -18 & 0 \\
\hline 1.3 & 1.2 & 1.0 & 1.2 & -17 & 0 \\
\hline 1.4 & 2.8 & 2.4 & 2.2 & 9 & 27 \\
\hline 1.5 & 2.0 & 2.2 & 2.2 & 0 & -9 \\
\hline 1.6 & 3.5 & 2.8 & 2.4 & 17 & 46 \\
\hline 1.7 & 2.9 & 2.7 & 2.2 & 23 & 32 \\
\hline 1.8 & 2.8 & 2.8 & 2.3 & 22 & 22 \\
\hline 1.9 & 3.0 & 2.7 & 2.2 & 23 & 36 \\
\hline 2.1 & 17.4 & 7.9 & 7.6 & 4 & 129 \\
\hline 2.2 & 17.8 & 8.0 & 7.4 & 8 & 141 \\
\hline 2.3 & 11.2 & 6.6 & 4.7 & 40 & 138 \\
\hline 2.4 & 12.0 & 6.7 & 4.6 & 46 & 161 \\
\hline 2.5 & 16.5 & 10.5 & 6.0 & 75 & 175 \\
\hline Всего & 95 & 58.2 & 47.2 & 23 & 101 \\
\hline
\end{tabular}

Здесь: $t_{B}, t_{D}, t_{E}$ - время, затраченное экспертом на решение комплекта задач соответственно вручную, в среде Derive 6 и в среде MS Excel.

Анализ таблицы 1 и диаграммы, представленной на рисунке 1 , говорит о том, что применение как математической системы Derive 6, так и табличного редактора MS Excel для автоматизации вычислений дает значительный выигрыш в затратах учебного времени по сравнению с расчетами вручную (более чем в 1,5 и в 2 раза соответственно).

В то же время использование электронных таблиц MS Excel для автоматизации рутинных действий дает больший выигрыш в затратах учебного времени по сравнению с системой Derive 6 (особенно при вычислении определителей большой размерности). 


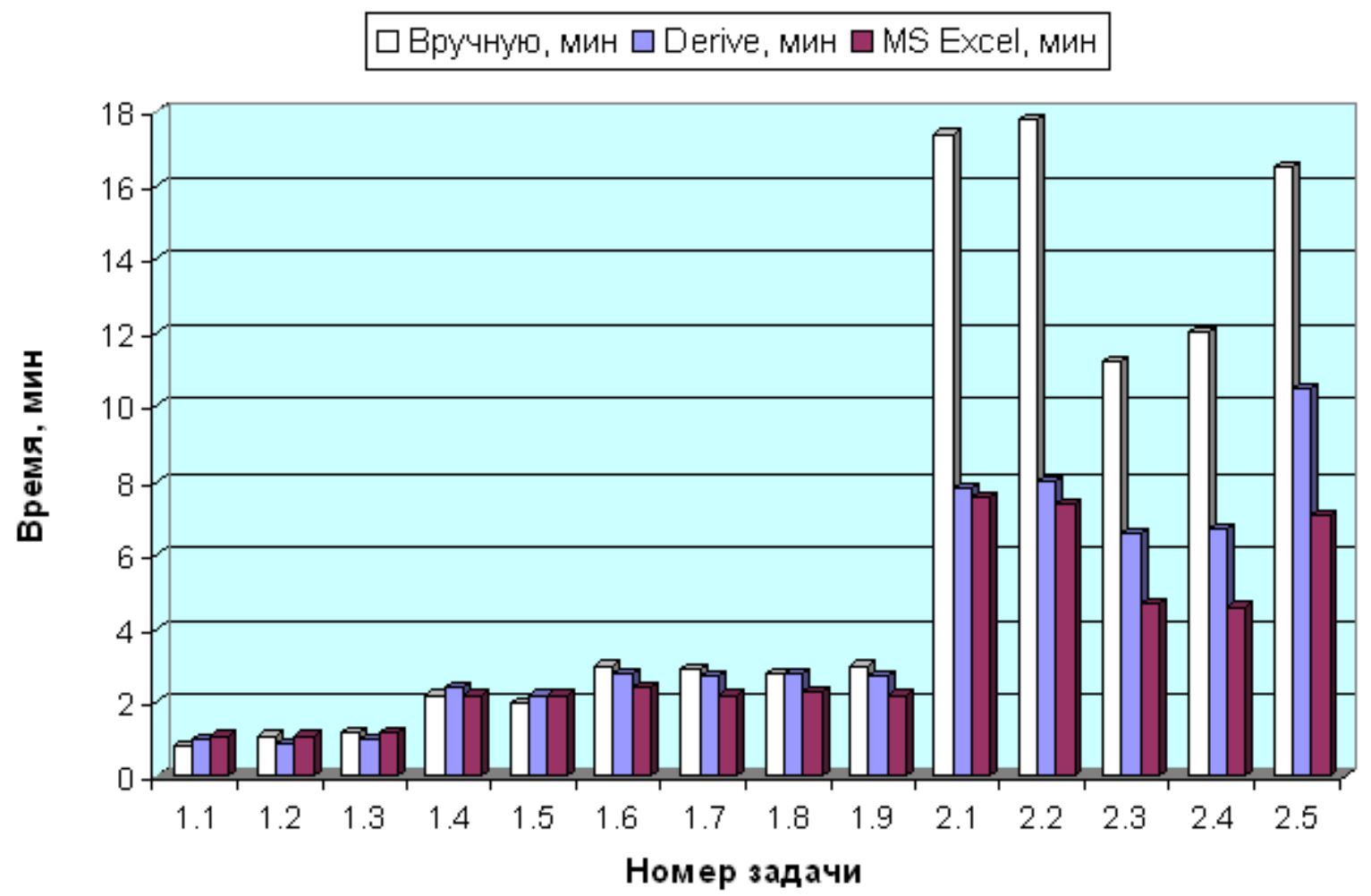

Рисунок 1. Время, затраченное экспертом на решение комплекта задач

Из проведенного анализа результатов первого этапа педагогического эксперимента следует, что в качестве программного обеспечения практического занятия «Вычисление определителей» табличный процессор MS Excel является более предпочтительным по сравнению с системой Derive 6.

На решение задач (за вычетом времени на вводную и заключительную части практического занятия) отводится примерно 70 минут учебного времени. За это время при проведении занятия в традиционной форме (без применения компьютеров) удается, как правило, решить семь задач: вычислить три определителя второго порядка, два определителя третьего порядка (методом Саррюса) и два определителя четвертого порядка (методом разложения по строке или столбцу). Метод элементарных преобразований вообще не рассматривается из-за нехватки учебного времени.

На решение комплекта задач с использованием MS Excel экспертом затрачено 47,2 минуты. Учитывая, что в школьном курсе информатики обучают работе с таблицами MS Excel и что с курсантами было проведено лабораторное занятие по освоению действий в среде табличного редактора, можно предположить, что, используя возможности MS Excel, за 70 минут учебного времени число задач, решаемых на данном практическом занятии можно существенно увеличить (ориентировочно с 7 до 14 штук) и при этом рассмотреть такой важный метод вычисления определителей, как метод элементарных преобразований.

Проверка этого предположения (гипотезы) и является содержанием второго проверочного этапа педагогического эксперимента (конструирующий эксперимент). На этом этапе в двух примерно равноценных по успеваемости учебных группах, контрольной и экспериментальной было проведено практическое занятие «Вычисление определителей» соответственно по традиционной методике и с применением MS Excel. Курсантам предлагались задачи описанного выше комплекта. В контрольной группе (традиционная методика) удалось решить семь задач комплекта заданий и лишь обозначить идею метода элементарных преобразований. В экспериментальной группе (с применением MS Excel) всеми курсантами были решены 13 заданий. Кроме того, хорошо успевающие курсанты смогли решить четырнадцатую задачу, вычислив определитель пятого порядка сведением его элементарными преобразованиями к определителю третьего порядка, который затем был вычислен автоматически с помощью встроенной функции.

Таким образом, применение редактора MS Excel позволило увеличить число решаемых на занятии задач с семи до тринадцати четырнадцати, т. е. практически в 2 раза. При этом курсанты экспериментальной группы в отличие от контрольной освоили также метод элементарных преобразований вычисления определителей. Все это является несомненной предпосылкой для повышения эффективности обучения курсантов вычислению определителей.

Итоговый (определяющий) этап педагогического эксперимента был реализован в форме проверки остаточных знаний курсантов контрольной и экспериментальной групп в конце семестра. Курсантам была предложена 30-и минутная 
самостоятельная работа, в которой требовалось вычислить один определитель третьего порядка по правилу Саррюса и один определитель четвертого порядка разложением по строке (столбцу). Результаты оценивания приведены в таблице 2 .

Результаты контроля остаточных знаний

Таблица 2

\begin{tabular}{|c|c|c|c|c|c|}
\hline $\begin{array}{l}\text { Количество } \\
\text { курсантов в группах }\end{array}$ & 2 & 3 & 4 & 5 & $\begin{array}{c}\text { Средний } \\
\text { балл }\end{array}$ \\
\hline $\begin{array}{c}\text { Контрольная группа } \\
\text { (20 человек) }\end{array}$ & $\begin{array}{c}3 \\
(15 \%)\end{array}$ & $\begin{array}{c}9 \\
(45 \%)\end{array}$ & $\begin{array}{c}6 \\
(30 \%)\end{array}$ & $\begin{array}{c}2 \\
(10 \%)\end{array}$ & 3,35 \\
\hline $\begin{array}{c}\text { Экспериментальная группа } \\
\text { (21 человек) }\end{array}$ & $\begin{array}{c}1 \\
(5 \%)\end{array}$ & $\begin{array}{c}4 \\
(19 \%)\end{array}$ & $\begin{array}{c}11 \\
(52 \%)\end{array}$ & $\begin{array}{c}5 \\
(24 \%)\end{array}$ & 3,95 \\
\hline
\end{tabular}

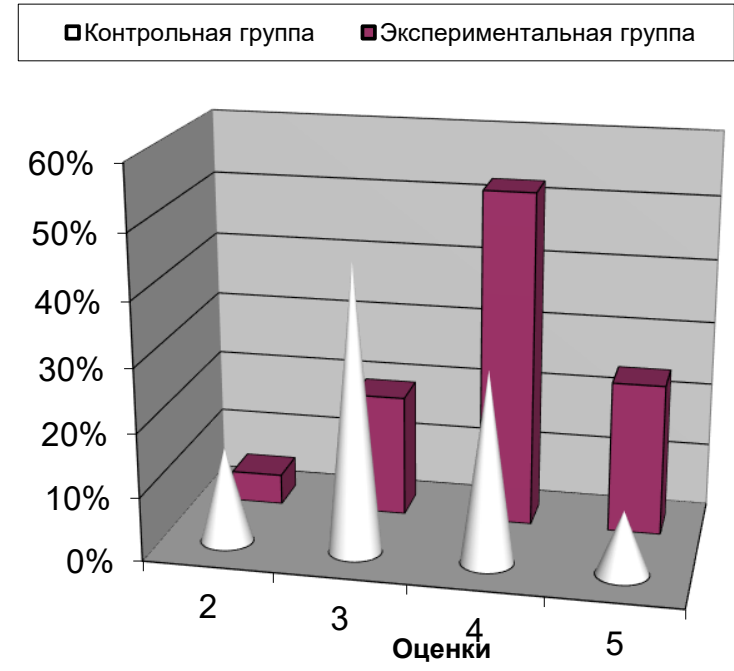

Рисунок 2. Результаты контроля остаточных знаний

Для количественной оценки эффективности обсуждаемой методики рассмотрим коэффициент успешности $K_{\text {ус }}$ и коэффициент качества $K_{\text {кач }}$ подготовки курсантов:

$$
K_{\text {ус }}=\frac{m}{n}, K_{\text {кач }}=\frac{k}{n}
$$

где $m$ - число положительных оценок, $k$ - число хороших и отличных оценок, $n$ - общее число оценок в группе.

$\mathrm{B}$ соответствии с таблицей 2 получим:

для контрольной группы $K_{\mathrm{yc}}=85 \%$, $K_{\text {кач }}=40 \%$;

для экспериментальной группы $K_{\text {ус }}=95 \%, K_{\text {кач }}=76 \%$.

Как видим, коэффициент успешности в экспериментальной группе по сравнению с контрольной выше на 10\%, а коэффициент качества выше на 36\%. Это позволяет сделать вывод, что предлагаемая методика проведения практического занятия «Вычисление определителей» с использованием табличного процессора MS Excel эффективна по критериям общей успешности и качественному показателю.
Таким образом, гипотеза о том, что автоматизация рутинных вычислений средствами табличного процессора MS Excel приведет к повышению эффективности обучения курсантов вычислению определителей, подтверждена педагогическим экспериментом.

\section{Литература:}

1. Паршин А. В. Телекоммуникационная видеосеть на базе персональных ЭВМ и ее применение в образовательном процессе. Журнал «Телекоммуникации», №6. - М.: Наука и технологии, 2003. - С. 56-61.

2. Паршин А.В., Гнездилов А.В., Лебедев А.В. Анализ возможностей математической системы Derive 6 по эффективной организации процесса обучения общим математическим и естественнонаучным дисциплинам в военном вузе. СРДР. Серия Б. Выпуск №75. - М.: ЦВНИ МО РФ, 2006. -53 c.

3. Паршин А.В. Математика. Основы автоматизированного вычислительного практикума. Учебное пособие - Воронеж: МО РФ, 2008. - 332 с.

4. Коровин В.Н. Учебная и методическая работа в высшем военно-учебном заведении. Воронеж: ВИРЭ, 2000. - 275 с. 